\title{
Identification of anti-proliferative kinase inhibitors as potential therapeutic agents to treat canine osteosarcoma
}

\begin{abstract}
Osteosarcoma is the most common primary bone tumour in dogs but various forms of therapy have not significantly improved clinical outcomes. As dysregulation of kinase activity is often present in tumours, kinases represent attractive molecular targets for cancer therapy. The purpose of this study was to identify novel compounds targeting kinases with the potential to induce cell death in a panel of canine osteosarcoma cell lines. The ability of 80 well-characterized kinase inhibitor compounds to inhibit the proliferation of four canine osteosarcoma cell lines was investigated in vitro. For those compounds with activity, the mechanism of action and capability to potentiate the activity of doxorubicin was further evaluated. The screening showed 22 different kinase inhibitors that induced significant antiproliferative effects across the four canine osteosarcoma cell lines investigated. Four of these compounds (RO 31-8220, 5-iodotubercidin, BAY 11-7082 and an erbstatin analog) showed significant cell growth inhibitory effects across all cell lines in association with variable induction of apoptosis. RO 31-8220 and 5-iodotubercidin showed the highest ability to potentiate the effects of doxorubicin on cell viability. In conclusion, the present study identified several potent kinase inhibitors targeting the PKC, CK1, PKA, ErbB2, mTOR and NF-əB pathways, which may warrant further investigations for the treatment of osteosarcoma in dogs.
\end{abstract}

Keyword: Canine; Cell lines; Kinase inhibitor; Osteosarcoma; Screening 